\title{
Special issue on fluorine-19 magnetic resonance: technical solutions, research promises and frontier applications
}

\author{
Sonia Waiczies ${ }^{1} \cdot$ Mangala Srinivas $^{2}$ - Ulrich Flögel ${ }^{3} \cdot$ Philipp Boehm-Sturm $^{4,5} \cdot$ Thoralf Niendorf $^{1}$
}

Published online: 7 February 2019

(c) European Society for Magnetic Resonance in Medicine and Biology (ESMRMB) 2019

Research on fluorine MR has been progressing at an impressive pace and yielding tangible results at the forefront of biomedical research. Several decades after its first documented application [1], the unprecedented opportunities of fluorine MRI in medical research remain intense and represent an area of increasing clinical interest. The challenges associated with fluorine MRI are also equally recognized, most notably the restrictively low detection limits and sensitivity boundaries. Nevertheless, fluorine MRI remains an extremely attractive method for following fluorinated labels that avoids the use of radioactive tracers. New developments from various research domains such as chemistry, physics, engineering and material sciences have joined forces to overcome these challenges and technical barriers. In fact, progress in fluorine MRI spans a very broad range of fields, from the chemistry of label and probe synthesis and production, through to biological and clinical applications, as well as physics, information technology and data science for image acquisition and processing [2].

The cutting-edge papers in this Special Issue illustrate new developments in the field of fluorine MRI from different research domains and demonstrate the versatility of these developments for clinical application.

Sonia Waiczies

Sonia.Waiczies@mdc-berlin.de

Mangala Srinivas

Mangala.Srinivas@gmail.com

Ulrich Flögel

floegel@uni-duesseldorf.de

Philipp Boehm-Sturm

philipp.boehm-sturm@ charite.de

Thoralf Niendorf

thoralf.niendorf@mdc-berlin.de

1 Berlin Ultrahigh Field Facility (B.U.F.F.), Max-DelbrueckCenter for Molecular Medicine in the Helmholtz Association, Berlin, Germany
One important focus of fluorine MRI research is the driving of technical developments surrounding MR signal acquisition and data processing, to lower the detection levels and boost sensitivity. Several innovative developments such as compressed sensing (CS) will ultimately be required to provide the high signal-to noise ratio (SNR) efficiency necessary for fluorine MRI. Controlling the fidelity of these methods will also be an essential task on the way to reaching the desired sensitivity for in vivo measurements. For instance, low SNR can lead to artifacts in CS reconstructions that reduce the image quality. This can be compensated by data resampling strategies that improve image quality and suppress background [3]. Quantification of the fluorine MR signal will also be essential for most of the conceivable applications [4-11]. Therefore, the necessary technologies such as $\mathrm{B}_{1}$ mapping to correct for non-uniform radiofrequency fields [12] or development of new radio frequency coil technologies $[13,14]$ must be available to make quantification of the fluorine MR signal feasible and accurate.

The design and synthesis of novel fluorine-rich molecular labels and imaging probes is another important domain in fluorine MRI research. Fluorinated probes are commonly designed with the goal of improving signal strength by increasing the number of fluorine atoms per molecule $[4$,

2 Department of Tumor Immunology, Radboud Institute for Molecular Life Sciences (RIMLS), Radboud University Medical Center (RadboudUMC), Nijmegen, The Netherlands

3 Experimental Cardiovascular Imaging, Molecular Cardiology, Heinrich Heine University, Düsseldorf, Germany

4 Department of Experimental Neurology and Center for Stroke Research Berlin, Charité - Universitätsmedizin Berlin, corporate member of Freie Universität Berlin, Humboldt-Universität zu Berlin, and Berlin Institute of Health, Berlin, Germany

5 Charité, Universitätsmedizin Berlin, NeuroCure Cluster of Excellence and Charité Core Facility 7T Experimental MRIs, Berlin, Germany 
15]. Such fluorinated probes have been particularly valuable for labeling cells to track them in vivo. In this special issue, a dissociation of the fluorescence and the fluorine label was shown signal over time, both in vitro and in vivo. This has a critical impact on the interpretation of long-term experiments validated by standard fluorescent techniques [6]. Fluorine-rich probes can also act as labels within microcapsules impregnated within cells for in vivo applications; e.g., microcapsules containing and protecting stem cells from immune rejection following transplantation [8].

One strategy in fluorine MR research is to manipulate the MR properties of the fluorinated probes to favor ideal conditions for MR signal acquisition. The complexation of fluorinated chelates with paramagnetic lanthanide ions leads to enhanced spin-lattice relaxation [16], thereby opening up the possibility for increasing SNR per unit time. Researchers have also designed and employed probes that prompt the desired MR signal following a trigger. The trigger is commonly a change in environmental conditions such as temperature [17], $\mathrm{pH}$ [18] or oxygenation state [7]. This has implications for several pathological situations. Perfluorocarbon nanoemulsions can determine changes in tissue oxygenation during vascular pathology, as shown in model of vascular cognitive impairment [7]. Functionally active crystal structures such as nickel complexes act as $\mathrm{pH}$ sensors [18], thereby positioning them as potential tumor markers for future in vivo studies. Investigating the relaxation properties of fluorinated drugs under different environmental conditions such as temperature [17] within the physiological range will also be an absolute requirement for studying drug distribution in vivo. Fluorination is a process in medicinal chemistry that is usually meant to improve pharmacological activity, but it does not preclude the use of fluorinated drugs as fluorine MRI probes. The MR activity of fluorinated drugs is an added benefit, above and beyond the expected enhancements in pharmacological activity, and will be an invaluable asset to study drug distribution and bioavailability [11].

The application of fluorine probes and fluorine MRI in (pre)clinical disease settings continues to expand, particularly in the area of diagnostics such as imaging of inflammation [5, 9], tissue oxygenation [7], and assessment of bile acid disorders [10]. This translational research is very encouraging en route to clinical applications and provides expanded knowledge into the distribution of the fluorinated probes in larger animals, particularly during pathology, and while using clinical scanners, thus advancing the opportunities of fluorine MRI for clinical application.

All research efforts and goals from these various domains are centered on the need for promoting fluorine MR signal to answer crucial questions in pathophysiology. Achieving this common goal will certainly require extra resourcesand the will to go there. The endeavor to take advantage of enabling technologies that go beyond standard tools, such as cutting-edge cryogenically cooled radio frequency coils and ultrahigh magnetic field strengths [19], will certainly require support from research institutions, funders and government bodies. Most of the efforts devoted to discovery and proof-of-principle research will benefit from an integration of these tools and technologies, to reach the maximum potential and realize the faraway visions and goals that will contribute to patient health care. The question as to whether the health system will eventually be able to adopt this stateof-the-art research into clinical settings will depend on the passion and motivation in our current research endeavors.

We hope that this issue will convey this dynamic vision and inspire you to also become pioneers in pushing the envelope of current technologies in fluorine MRI to be able to answer challenging and important biological questions in the future.

Guest Editors

Sonia Waiczies

Mangala Srinivas

Ulrich Flögel

Philipp Boehm-Sturm

Thoralf Niendorf

Editor

David G. Norris

\section{Compliance with ethical standards}

Conflict of interest The authors declare that they do not have a conflict of interest with the exception that Thoralf Niendorf is CEO and founder of MRI.TOOLS GmbH, Berlin, Germany.

Ethical standards This editorial does not contain any studies with human participants or animals performed by any of the authors with the exception of references to previously published work included in this editorial.

\section{References}

1. Holland G, Bottomley P, Hinshaw W (1977) ${ }^{19} \mathrm{~F}$ magnetic resonance imaging. J Magn Reson 28:133-136

2. Flogel U, Ahrens E (2016) Fluorine magnetic resonance imaging. Pan Stanford, New York

3. Kampf T, Sturm VJF, Basse-Lüsebrink TC, Fischer A, Buschle LR, Kurz FT, Schlemmer HP, Ziener CH, Heiland S, Bendszus M, Pham M, Stoll G, Jakob PM (2018) Improved compressed sensing reconstruction for ${ }^{19} \mathrm{~F}$ magnetic resonance imaging. Magn Reson Mater Phy. https://doi.org/10.1007/s10334-018-0729-1

4. Liu X, Jiang Z-X, Yu BY, Jeong E-K (2018) Study of kinetics of ${ }^{19}$ F-MRI using a fluorinated imaging agent (19FIT) on a 3T clinical MRI system. Magn Reson Mater Phy. https://doi.org/10.1007/ s10334-018-0707-7 
5. Makela AV, Foster PJ (2018) Preclinical ${ }^{19} \mathrm{~F}$ MRI cell tracking at 3 Tesla. Magn Reson Mater Phy. https://doi.org/10.1007/s1033 4-018-0715-7

6. Bouvain P, Flocke V, Krämer W, Schubert R, Schrader J, Flögel $\mathrm{U}$, Temme S (2018) Dissociation of ${ }^{19} \mathrm{~F}$ and fluorescence signal upon cellular uptake of dual-contrast perfluorocarbon nanoemulsions. Magn Reson Mater Phy. https://doi.org/10.1007/s1033 4-018-0723-7

7. Khalil AA, Mueller S, Foddis M, Mosch L, Lips J, Przesdzing I, Temme S, Flögel U, Dirnagl U, Boehm-Sturm P (2018) Longitudinal ${ }^{19} \mathrm{~F}$ magnetic resonance imaging of brain oxygenation in a mouse model of vascular cognitive impairment using a cryogenic radiofrequency coil. Magn Reson Mater Phy. https://doi. org/10.1007/s10334-018-0712-X

8. Wang G, Fu Y, Shea SM, Hegde SS, Kraitchman DL (2018) Quantitative CT and ${ }^{19} \mathrm{~F}-\mathrm{MRI}$ tracking of perfluorinated encapsulated mesenchymal stem cells to assess graft immunorejection. Magn Reson Mater Phy. https://doi.org/10.1007/s10334-018-0728-2

9. Rothe M, Jahn A, Weiss K, Hwang J-H, Szendroedi J, Kelm M, Schrader J, Roden M, Flögel U, Bönner F (2018) In vivo ${ }^{19} \mathrm{~F}$ MR inflammation imaging after myocardial infarction in a large animal model at 3 T. Magn Reson Mater Phy. https://doi.org/10.1007/ s10334-018-0714-8

10. Raufman J-P, Metry M, Felton J, Cheng K, Xu S, Polli J (2018) A ${ }^{19} \mathrm{~F}$ magnetic resonance imaging-based diagnostic test for bile acid diarrhea. Magn Reson Mater Phy. https://doi.org/10.1007/ s10334-018-0713-9

11. Sitter B (2019) Urinary detection of corticosteroid in topical treatment of skin disease by ${ }^{19} \mathrm{~F}$ MRS. Magn Reson Mater Phy. https ://doi.org/10.1007/s10334-018-00734-y

12. Vernikouskaya I, Pochert A, Lindén M, Rasche V (2018) Quantitative ${ }^{19} \mathrm{~F}$ MRI of perfluoro-15-crown-5-ether using uniformity correction of the spin excitation and signal reception. Magn Reson Mater Phy. https://doi.org/10.1007/s10334-018-0696-6

13. Villa-Valverde P, Rodríguez I, Padró D, Benito M, GarridoSalmon CE, Ruiz-Cabello J (2019) A dual ${ }^{1} \mathrm{H} /{ }^{19} \mathrm{~F}$ birdcage coil for small animals at 7 Tesla MRI. Magn Reson Mater Phy. https ://doi.org/10.1007/s10334-018-00733-Z

14. Park BS, Ma G, Koch WT, Rajan SS, Mastromanolis M, Lam J, Sung K, McCright B (2018) Improvement of ${ }^{19} \mathrm{~F}$ MR image uniformity in a mouse model of cellular therapy using inductive coupling. Magn Reson Mater Phy. https://doi.org/10.1007/s1033 4-018-0693-9

15. Jirak D, Galisova A, Kolouchova K, Babuka D, Hruby M (2018) Fluorine polymer probes for magnetic resonance imaging: quo vadis? Magn Reson Mater Phy. https://doi.org/10.1007/s1033 4-018-0724-6

16. Herynek V, Martinisková M, Bobrova Y, Gálisová A, Kotek J, Hermann P, Koucký F, Jirák D, Hájek M (2018) Low-molecularweight paramagnetic ${ }^{19} \mathrm{~F}$ contrast agents for fluorine magnetic resonance imaging. Magn Reson Mater Phy. https://doi.org/10.1007/ s10334-018-0721-9

17. Prinz C, Delgado PR, Eigentler TW, Starke L, Niendorf T, Waiczies S (2018) Toward (19)F magnetic resonance thermometry: spin-lattice and spin-spin-relaxation times and temperature dependence of fluorinated drugs at 9.4 T. Magn Reson Mater Phy. https://doi.org/10.1007/s10334-10018-10722-10338

18. Xie D, Ohman LE, Que EL (2018) Towards Ni(II) complexes with spin switches for ${ }^{19} \mathrm{~F}$ MR-based $\mathrm{pH}$ sensing. Magn Reson Mater Phy. https://doi.org/10.1007/s10334-018-0698-4

19. Waiczies S, Rosenberg JT, Kuehne A, Starke L, Delgado PR, Millward JM, Prinz C, Dos Santos Periquito J, Pohlmann A, Waiczies H, Niendorf T (2018) Fluorine-19 MRI at 21.1 T: enhanced spinlattice relaxation of perfluoro-15-crown-5-ether and sensitivity as demonstrated in ex vivo murine neuroinflammation. Magn Reson Mater Phy. https://doi.org/10.1007/s10334-10018-10710-z

Publisher's Note Springer Nature remains neutral with regard to jurisdictional claims in published maps and institutional affiliations. 